\title{
THE MASS ASSEMBLY HISTORY OF SPHEROIDAL GALAXIES: DID NEWLY FORMED SYSTEMS ARISE VIA MAJOR MERGERS?
}

\author{
Kevin Bundy, ${ }^{1}$ Tommaso Treu, ${ }^{2}$ and Richard S. Ellis ${ }^{3}$ \\ Received 2007 March 13; accepted 2007 June 27; published 2007 July 27
}

\begin{abstract}
We examine the properties of a morphologically selected sample of $0.4<z<1.0$ spheroidal galaxies in the GOODS fields in order to ascertain whether or not their increase in abundance with time arises primarily from major mergers. To address this question, we determine the scaling relations between the dynamical mass $M_{\mathrm{dyn}}$, determined from stellar velocity dispersions, and the stellar mass $M_{*}$, determined from optical and infrared photometry. We exploit these relations across the larger sample for which we have stellar masses in order to construct the first statistically robust estimate of the evolving dynamical mass function (MF) over $0<z<1$. The trends observed match those seen in the stellar mass functions of Bundy et al. regarding the top-down growth in the abundance of spheroidal galaxies. By referencing our dynamical masses to the halo virial mass, $M_{\mathrm{vir}}$, we compare the growth rate in the abundance of spheroidals to that predicted by the assembly of dark matter halos. Our comparisons demonstrate that major mergers do not fully account for the appearance of new spheroidals since $z \sim 1$ and that additional mechanisms, such as morphological transformations, are required to drive the observed evolution.

Subject headings: galaxies: elliptical and lenticular, cD — galaxies: evolution galaxies: kinematics and dynamics — galaxies: luminosity function, mass function

Online material: color figure
\end{abstract}

\section{INTRODUCTION}

Substantial progress has been made in characterizing the primarily old, red, and passively evolving stellar populations of spheroidal galaxies (defined here to include elliptical and lenticulars) from $z \simeq 1$ to the present day (see Renzini 2006 for a review). Their evolving abundance contains valuable clues to the processes by which they form as well as insight into the history of cosmic mass assembly. In recent years, many surveys have converged on a broad evolutionary pattern in which it is claimed that galaxies with the largest stellar masses evolve predominantly into spheroidals by $z \gtrsim 1$ with little subsequent growth. Meanwhile, intermediate and lower mass systems continue to form at later epochs (e.g., Bundy et al. 2005, 2006; Tanaka et al. 2005; Franceschini et al. 2006; Borch et al. 2006; Pannella et al. 2006; Cimatti et al. 2006; Abraham et al. 2007).

Some authors (e.g., van Dokkum 2005) have questioned the absence of growth among massive galaxies, pointing to the high rate of tidal features in local examples, indicative of recent merging. It is likewise argued (Drory et al. 2004; van der Wel et al. 2006; Maraston et al. 2006; Kannappan \& Gawiser 2007) that stellar masses derived from optical-infrared photometry may suffer biases that lead to large uncertainties in the inferred growth rate. Beyond such concerns, interpreting the observed evolution in the context of the $\Lambda \mathrm{CDM}$ paradigm necessitates appealing to potentially uncertain semianalytic models. Clearly what is needed is a way to connect observations of galaxy growth directly to the assembly history of the halos in which they reside.

Spheroidal galaxies offer us particular advantages in this regard. First, because of their regular mass profiles, spectroscopic observations can be used to infer the dynamical mass, bypassing luminous quantities. Second, because of their pres-

\footnotetext{
${ }^{1}$ Reinhardt Fellow, Department of Astronomy and Astrophysics, University of Toronto, 50 St. George Street, Room 101, Toronto, ON M5S 3H4, Canada.

${ }^{2}$ Alfred P. Sloan Research Fellow, Department of Physics, University of California, Santa Barbara, CA 93106-9530.

${ }^{3}$ California Institute of Technology, MS 105-24, 1201 East California Boulvard, Pasadena, CA 91125.
}

sure-supported dynamical configurations, they are expected to result, for the most part, from mergers (e.g., Toomre 1977; Springel et al. 2005a). Accordingly, their evolving abundances can be compared directly to the expected assembly history of dark matter halos. In this Letter we develop a technique to estimate dynamical and halo masses for a large sample of field spheroidals and use it to interpret their evolution in the context of halo assembly as predicted in numerical simulations. We assume $\Omega_{M}=0.3, \Omega_{\Lambda}=0.7$, and $H_{0}=70 h_{70} \mathrm{~km} \mathrm{~s}^{-1} \mathrm{Mpc}^{-1}$.

\section{OBSERVATIONS AND MASS ESTIMATES}

The dynamical mass (hereafter $M_{\mathrm{dyn}}$ ) measurements used in this Letter are based on the analysis presented in Treu et al. (2005), to which we refer for details. Briefly, this sample comprises 165 field visually classified spheroidals selected from ACS imaging in the northern GOODS field to a magnitude limit of $z_{\mathrm{AB}}<22.5$ and studied with DEIMOS on Keck II. A subset of 125 galaxies have reliable stellar velocity dispersions, stellar masses, and surface photometry fits, which provide $B$-band effective radii, $R_{e, B}$ (see Treu et al. 2005). Dynamical masses were derived using the formula $M_{\mathrm{dyn}}=K_{v} \sigma^{2} R_{e, B} / G$, where $K_{v}$ is the virial coefficient and $\sigma$ is the central velocity dispersion, obtained by increasing the observed dispersion by $10 \%$ to correct to the standard circular aperture of diameter $R_{e, B} / 4$. Detailed modeling of fiber as well as three-dimensional spectroscopy of nearby ellipticals support the use of this equation (Padmanabhan et al. 2004; Cappellari et al. 2006), with little uncertainties due to the overall flatness of the velocity dispersion profile.

With the kinematic sample defined in this way, the investigation that follows is based on the much larger sample of galaxies with stellar masses (hereafter $M_{*}$ ) in both GOODS fields with $z_{\mathrm{AB}}<$ 22.5 (Bundy et al. 2005). We refer to Bundy et al. (2005) for further details but summarize the key features. For $0.4<z<$ 1.0, spectroscopic redshifts for 633 galaxies from the Keck Team Redshift Survey (Wirth et al. 2004) were supplemented with 695 determined using photometric techniques (see Bundy et al. 2005). The full sample of morphologically selected spheroidals includes 
393 sources. Using deep $K_{s}$-band imaging obtained at Palomar Observatory for GOODS-N and publicly available data in GOODS-S, stellar masses were estimated assuming a Chabrier (Chabrier 2003) initial mass function (IMF), using the code described in Bundy et al. (2006). For galaxies with photometric redshifts, an additional 0.3 dex is added in quadrature to the base uncertainty, which is typically 0.2 dex (Bundy et al. 2005, 2006).

As discussed in Bundy et al. (2005), the GOODS morphological sample is complete in the $K_{s}$ band but becomes incomplete for very red galaxies below a certain stellar mass limit as a result of the $z_{\mathrm{AB}}<22.5$ limit (see their Fig. 4). By considering synthetic spectral energy distributions representing red (in $z-$ $K_{s}$ color) stellar populations, we determine mass limits of $\log \left(M_{\text {limit }} / M_{\odot}\right)=[9.8,10.4,10.8]$ for redshifts $z=[0.4,0.7$, 1.0], respectively. The completeness limits of the MFs below are determined at the near edge of each redshift interval. When comparing $M_{*}$ to $M_{\mathrm{dyn}}$, we employ the more strict, volume-limited limits at the far edge of each interval (see discussion in Bundy et al. 2006).

\section{LINKING DYNAMICAL MASSES AND STELLAR MASSES}

We begin by restricting our analysis to those 125 spheroidals in GOODS-N for which both $M_{\mathrm{dyn}}$ and $M_{*}$ measurements are available. The aim is to develop scaling relations that can be applied to the larger GOODS-N/S sample. For local galaxies, a fairly tight correlation of the form $M_{\mathrm{dyn}}=\left(M_{*}\right)^{\alpha}$ has been observed by several authors (e.g., Padmanabhan et al. 2004; Cappellari et al. 2006; Gallazzi et al. 2006). Gallazzi et al. (2006) exploited a local sample of early-type systems selected by concentration, and they find $\alpha=1.28 \pm 0.03$ - the zero point is not explicitly solved for. In this work, we adopt a zero point such that $M_{\text {dyn }} \equiv M_{*}$ at $10^{11} M_{\odot}$, and we also apply it to the Gallazzi et al. (2006) relation. This choice corresponds to $K_{v}=4.8$ and 6.6 at $0.4<z<0.7$ and $0.7<z<1.0$, respectively, and is motivated by the fact that the $K_{v}$ depends on the mass density profile and, most importantly, on the spectroscopic aperture used to measure $\sigma$. Since the aperture is effectively redshift-dependent (the slit width is fixed at $1^{\prime \prime}$ ), assuming a constant $K_{v}$ could introduce a spurious redshift dependency. Similarly, while there is no evidence that the density profile evolves for high-mass spheroidals (e.g., Koopmans et al. 2006), potential evolution could mimic changes in the MF if $K_{v}$ were held fixed. More detailed studies are needed in order to determine the normalization independently.

We examine the $M_{\mathrm{dyn}}-M_{*}$ relation at $0.4<z<0.7$ and $0.7<$ $z<1.0$ in Figure 1, restricting the comparison to systems above our completeness limit; the best-fit values for $\alpha$ are also indicated. Our observed slopes are formally consistent with those of Gallazzi et al. (2006), $1.28 \pm 0.03$ at $z=0$, and Rettura et al. (2006), $1.25 \pm 0.05$ at $z \sim 1$ (di Serego Alighieri et al. 2005), although some differences may be expected as a result of sample selection and fiber- versus slit-based measures of velocity dispersion. The fact that $\alpha>1$ reflects the "tilt" of the fundamental plane (e.g., Faber et al. 1987; Ciotti et al. 1996; Renzini 2006) and is consistent with nonhomology (e.g., Trujillo et al. 2004), including an increasing dark matter fraction within $R_{e}$ at higher masses (e.g., Padmanabhan et al. 2004; Gallazzi et al. 2006; Bolton et al. 2007). With regard to what follows, the key result in Figure 1 is that $\alpha$ shows no measurable evolution across our sample. In $\S 4$, we will show that this leads to dynamical MFs that behave similarly to their stellar mass counterparts. For consistency, final $M_{\mathrm{dyn}}$ estimates for galaxies in the full GOODS sample are determined using the value of $\alpha$ determined for the two redshift bins, and the final

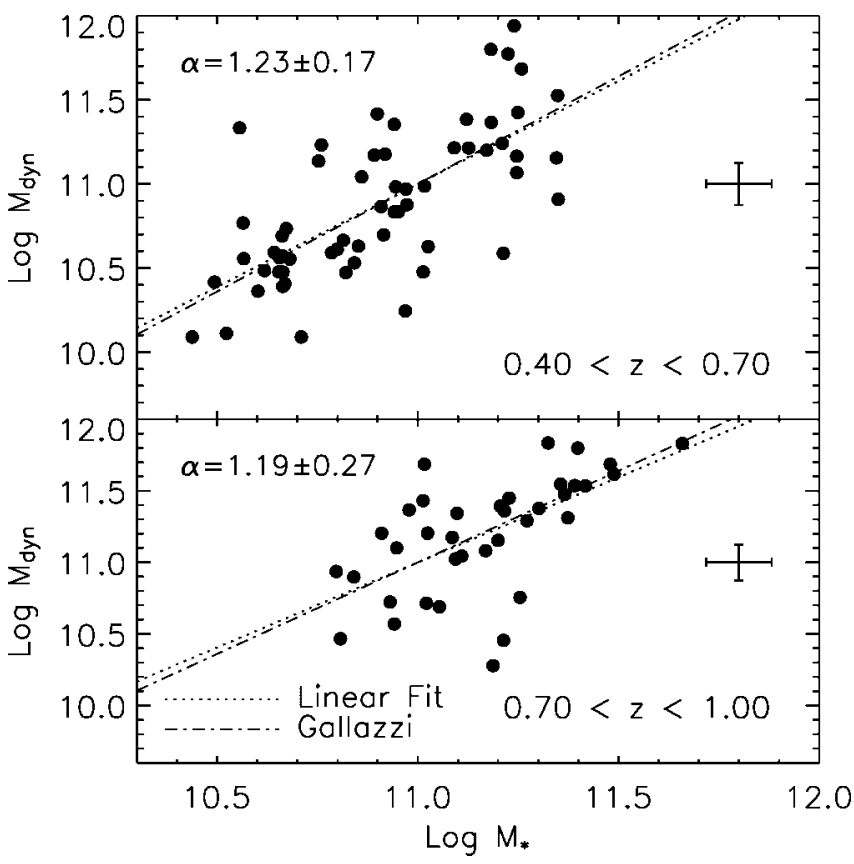

FIG. 1.-Correlation between $M_{\mathrm{dyn}}$ and $M_{*}$. The dotted line illustrates the fitted slope, $\alpha$, which is also indicated in the top left corner. A typical error bar is shown, and the relation found by Gallazzi et al. (2006) is also plotted. The normalization in each panel has been set to the same scale, as described in the text.

uncertainty in $M_{\mathrm{dyn}}$ is taken to be the sum in quadrature of the uncertainty in $M_{*}$ and the scatter in Figure 1.

\section{THE DYNAMICAL MASS FUNCTION}

Using the $M_{\text {dyn }}$ estimates derived above for the full GOODS sample, we now construct the dynamical MF and compare it with its stellar mass counterpart in the top two panels of Figure 2. We use the $1 / V_{\max }$ estimator and closely follow the method described in Bundy et al. (2005). The filled circles within the gray shading show the stellar MF for the full sample, while the crosses indicate the full stellar MF that results when the magnitude limit is relaxed to $z_{\mathrm{AB}}<23.5$. The bottom panel of Figure 2 shows the differential growth derived across the two redshift bins.

Figure 2 demonstrates consistency between the stellar MFs and the dynamical MFs. Although our adopted zero point in the $M_{\text {dyn }}$ relations ensures a similar vertical normalization, the application of our scaling relations does not mean that both MFs should have the same shape. Indeed, small differences are noticeable in our highest mass bin. The good agreement results in large part from the fact that the $M_{\mathrm{dyn}}-M_{*}$ relation does not vary significantly across our sample. Had a variation in $\alpha$ with redshift been present in Figure 1, the resulting dynamical MFs would evolve with respect to the stellar MFs. The fact that the two agree validates earlier stellar mass estimates at $z \sim 1$ and the top-down growth in the abundance of spheroidals derived from those measures.

\section{TESTING THE ROLE OF MERGERS}

We now turn to the key question that our analysis can address: Is the rising abundance with time of spheroidal galaxies consistent with that predicted by the growth of merging dark matter halos? To answer this question, we adopt a simple, observationally motivated model to connect $\sigma$ and $M_{*}$ to the virial mass, $M_{\text {vir }}$, of the host halos. Lensing studies of early-type galaxies at $z \approx 0.2$ (see Gavazzi et al. 2007 and references therein) show that the total mass density profile of spheroidal halos is nearly isothermal within 


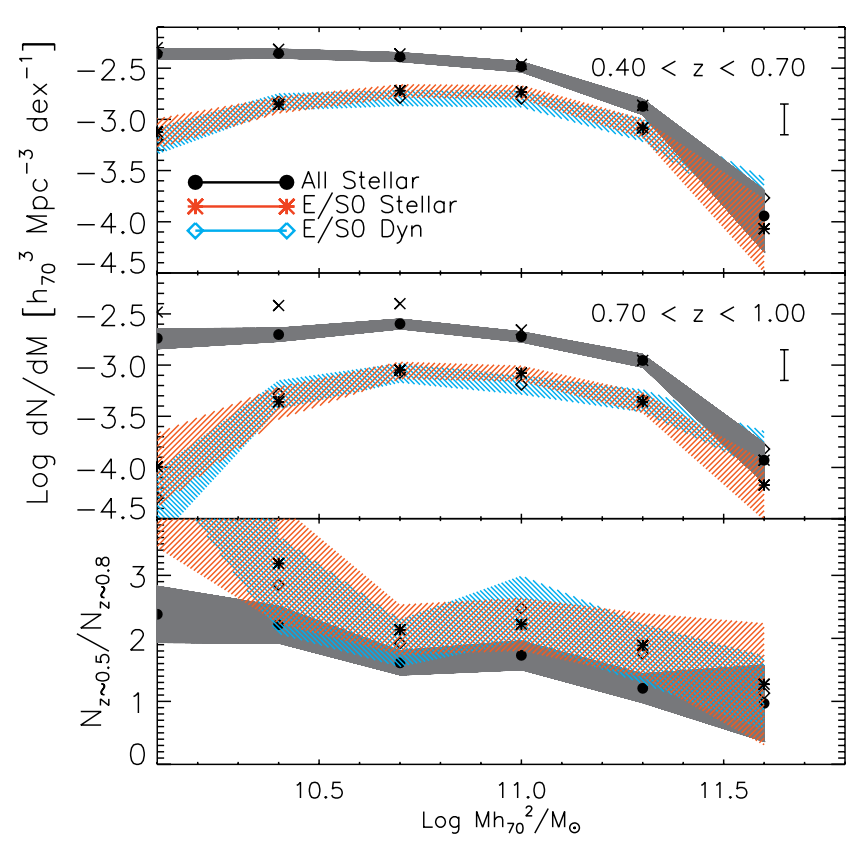

FIG. 2.-Comparison of evolving dynamical and stellar mass functions. The top two panels show the MFs in two redshift intervals, while the bottom panel illustrates the differential growth between them. The filled circles within the gray shading trace the stellar mass function of the full (all types) GOODS $z_{\mathrm{AB}}<22.5$ sample, while the crosses denote the equivalent $z_{\mathrm{AB}}<23.5$ sample. Asterisks within the light red shading trace the stellar MF of the spheroidal component, while the open diamonds within the blue shading indicate the dynamical MF. The fact that the observed increase with time in the spheroidal MFs is more significant than the increase observed for the full sample (bottom panel) shows that this result is robust to potential incompleteness. Isolated error bars indicate the estimated uncertainty from cosmic variance.

the halo scale radius, $r_{s}$. We therefore assume a mass profile that is isothermal for $r \leqq r_{s}$ and follows a $\rho \propto r^{-3}$ profile (NFW; Navarro et al. 1997) for $r \gtrsim r_{s}$. We note that the $\rho \propto r^{-3}$ behavior at large radii $\left(r \sim r_{\text {vir }}\right)$ is consistent with non-NFW scaling laws that can disagree instead at small radii (e.g., Graham et al. 2006). The mass profile assumed here is given by $\rho(r)=$ $\sigma^{2}\left[2 \pi G r^{2}\left(1+r / \gamma r_{s}\right)\right]^{-1}$, where $\sigma$ is the observed central velocity dispersion and we have introduced the scaling parameter, $\gamma$, which can be used to tune the profile shape to observations. By comparing this mass profile to that of Gavazzi et al. (2007), we find $\gamma=$ 12-future observations will constrain $\gamma$ as a function of redshift and mass. We define $M_{\mathrm{vir}}=(4 \pi / 3) \Delta(z) \rho_{c} c^{3} r_{s}^{3}$, where $\rho_{c}$ is the cosmic critical density, $\Delta(z)$ is the overdensity parameter (see Bryan \& Norman 1998), and $r_{\mathrm{vir}}=c r_{s}$. The concentration, $c$, is assumed to the follow the relation found in simulations, $c=$ $9(1+z)^{-1}\left[M_{\mathrm{vir}} /\left(8.12 \times 10^{12} h^{-1} M_{\odot}\right)\right]^{-0.14}$ (Bullock et al. 2001). Using this model and the relations above, we solve for $M_{\text {vir }}$ as a function of velocity dispersion and find

$$
\log M_{\mathrm{vir}}=A_{\sigma} \log \sigma_{200}+B_{\sigma}+C_{\sigma}(1+z)
$$

where $M_{\text {vir }}$ is in units units of $h_{70}^{-1} M_{\odot}, \sigma_{200}$ is the velocity dispersion in units of $200 \mathrm{~km} \mathrm{~s}^{-1}$, and the fit parameters are given by $A_{\sigma}=3.1, B_{\sigma}=13.2$, and $C_{\sigma}=-0.3$. Equation (1) follows from the model described above, and so random uncertainties on the fitting parameters are negligible. However, systematic errors resulting from tuning this model to observations should reflect the uncertainty found by Gavazzi et al. (2007) of 0.2 dex. Using our kinematic sample, for which we measure both $\sigma$ and $M_{*}$, we can

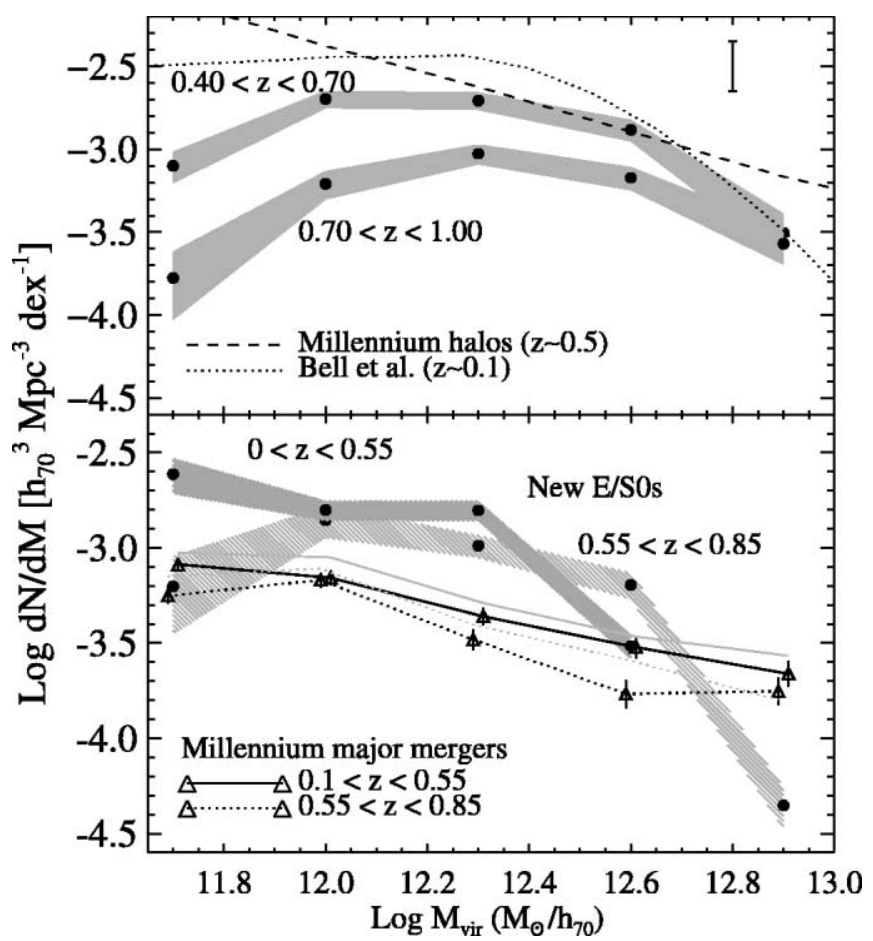

FIG. 3.-Evolving virial mass functions of halos hosting spheroidals. The top panel shows MFs in two $z$-bins. The local MF estimated from Bell et al. (2003) is indicated by the dotted line. The dashed line shows the total MF of predicted dark matter halos, and the isolated error bar (top right) denotes the cosmic variance uncertainty. The bottom panel shows the MF of newly formed spheroidals determined by subtracting the MFs (top panel) in adjacent redshift bins. Overplotted are two halo merger scenarios based on the Millennium simulation, integrated over the redshift ranges indicated. The black solid and dotted lines with the open triangles trace $>1: 3$ mergers, while the gray lines (without symbols) denote such mergers with a $\sim 0.5 \mathrm{Gyr}$ time delay. [See the electronic edition of the Journal for a color version of this figure.]

also directly compare $M_{\text {vir }}$ and $M_{*}$, finding the following relation:

$$
\log M_{\mathrm{vir}}=A_{*} \log M_{* 11}+B_{*}+C_{*}(1+z)
$$

where a Chabrier IMF was assumed as before and $M_{* 11}$ is in units of $10^{11} h_{70}^{-2} M_{\odot}$. The best-fitting parameters are $A_{*}=1.0 \pm$ $0.2, B_{*}=12.83 \pm 0.11 \pm \sigma_{B \text {, sys }}$, and $C_{*}=-0.2 \pm 0.1$, where $\sigma_{B \text {, sys }}=0.25$ dex and accounts for the systematic uncertainty in equation (1) (0.2 dex) as well as the uncertainty in the stellar IMF (0.15 dex). Equations (1) and (2) are applicable for galaxies with $\log \left(M_{*} / M_{\odot}\right) \approx 10-12$ and $\sigma \approx 90-300 \mathrm{~km} \mathrm{~s}^{-1}$. Equation (2) gives values of $M_{\mathrm{vir}} / M_{*}=30_{-15}^{+25}$, consistent with Mandelbaum et al. (2005) and Gavazzi et al. (2007). We note that our marginally nonzero value of $C_{*}$, if confirmed, implies a slight increase with time of $M_{\mathrm{vir}} / M_{*}(\sim 30 \%)$ over $0.4<z<1.0$ that is consistent with halo growth (see discussion in Conroy et al. 2007) and independent evidence that stellar mass assembly is mostly completed by $z \approx$ 1 (e.g., Treu et al. 2005). We also note that virial mass and stellar mass turn out to be approximately in linear proportion within this mass range. Studies with a larger dynamical range in stellar masses and velocity dispersion, and more weak-lensing measurements, are needed to measure the slope with higher precision and detect departures from linearity, expected as a result of varying star formation efficiency with halo mass.

Using equation (2), we estimate halo virial masses for our sample and compare the resulting virial MFs in the top panel of Figure 3. The dashed line is the total halo mass function 
predicted at $z \approx 0.5$ (it evolves little over $0<z<1.0$ ) using the online Millennium Simulation Database ${ }^{4}$ (Springel et al. 2005b) and adjusted in number density by +0.1 dex to be consistent with the spheroidal MFs (this adjustment is within the expected cosmic variance of $0.15 \mathrm{dex}$ ). The dotted line shows a local $\left(z \approx 0.1\right.$ ) estimate constructed by converting the $M_{*}$ MF for early types (based on concentration, $C \geq 2.6$ ) measured by Bell et al. (2003) into a virial MF using equation (2). Adjustments of +0.1 dex were also applied to the local number density and the local mass scale to match the GOODS sample at high masses. Such corrections are likely needed as a result of cosmic variance, differing selection and mass estimate methods, and photometric uncertainties. Thus, comparisons between our results and the local MF must be done with caution.

The top panel of Figure 3 reveals a similar pattern of evolution as seen in Figure 2. A useful interpretation of this evolution is given in the bottom panel of Figure 3, which plots the $\log$ difference $[\log (d N / d M)]$, thereby displaying the halo MF of new spheroidals that have appeared over the time interval separating the two redshift windows and providing a way to compare with the incidence of halo mergers. We use the MPAHalo milliMillennium database to identify halos that have experienced a recent merger, at which point two previously separate halos or subhalos become a single halo. Halo merging often occurs several gigayears after halo accretion, defined by the point at which the two progenitor halos first become associated with the same friendsof-friends group. Because the smaller progenitor halo may experience significant tidal stripping after halo accretion but before merging (see Gao et al. 2004), we take the merger mass ratio to be the initial ratio before halo accretion.

After integrating over the two time intervals that separate our observations, we overplot two merging scenarios in the bottom panel of Figure 3 (the +0.1 dex vertical offset is also applied). It is a striking result that major halo mergers (mass

\footnotetext{
${ }^{4}$ The "milli-Millennium" database covers a volume of $7 \times 10^{5} h_{70}^{-3} \mathrm{Mpc}^{3}$, nearly 4 times larger than our largest sampled volume at $0.7<z<0.4$ (see http://www.mpa-garching.mpg.de/millennium).
}

ratios greater than $1: 3$, indicated by the black solid and dotted lines) cannot account for the rise of spheroidal systems over the two epochs probed here. The light gray lines (without symbols) show that this is still the case when a $\sim 0.5$ Gyr delay is added to the merger timescale (as suggested by expected differences in the galaxy and halo merger times).

\section{DISCUSSION}

By constructing dynamical mass functions for 393 field spheroidals and linking those mass estimates to the halos in which they reside, we have found a significant result in the bottom panel of Figure 3: major merging of dark matter halos as described by $\Lambda \mathrm{CDM}$ does not occur frequently enough to explain the observed increase in the mass function of spheroidal galaxies. A check made by appealing to the semianalytic model of de Lucia \& Blaizot (2007) shows a similar result when a galaxy's stellar mass is used to define recent mergers and even when $M_{*}$ is used as the "accounting variable."

If merging is not the only mechanism that produces newly formed spheroidals since $z \sim 1$, other physical processes likely play a significant role. These may include mechanisms for transforming disk and irregular galaxies into relaxed spheroidals, for example, secular bulge growth accompanied by disk fading. Indeed, recent semianalytic models (e.g., Croton et al. 2006; Bower et al. 2006; de Lucia \& Blaizot 2007) have required prescriptions for such mechanisms, in addition to mergers, to match the local abundance of spheroidals. More work is needed to understand these processes and their role in morphological evolution.

We are very grateful to Simon White and Volker Springel for help in deriving results from the Millennium Run Database, and we also thank Raphael Gavazzi, Andrew Benson, Phil Hopkins, and Karl Glazebrook for useful discussions. R. S. E. acknowledges the hospitality of Ray Carlberg at the University of Toronto during a sabbatical visit, and T. T. acknowledges support from a Sloan Research Fellowship.

\section{REFERENCES}

Abraham, R. G., et al. 2007, ApJ, in press (astro-ph/0701779)

Bell, E. F., McIntosh, D. H., Katz, N., \& Weinberg, M. D. 2003, ApJ, 585, L117 Bolton, A. S., Burles, S., Treu, T., Koopmans, L. V. E., \& Moustakas, L. A. 2007, ApJL, submitted (astro-ph/0701706)

Borch, A. et al. 2006, A\&A, 453, 869

Bower, R. G., et al. 2006, MNRAS, 370, 645

Bryan, G. L., \& Norman, M. L. 1998, ApJ, 495, 80

Bullock, J. S., Kolatt, T. S., Sigad, Y., Somerville, R. S., Kravtsov, A. V., Klypin, A. A., Primack, J. R., \& Dekel, A. 2001, MNRAS, 321, 559

Bundy, K., Ellis, R. S., \& Conselice, C. J. 2005, ApJ, 625, 621

Bundy, K., et al. 2006, ApJ, 651, 120

Cappellari, M., et al. 2006, MNRAS, 366, 1126

Chabrier, G. 2003, PASP, 115, 763

Cimatti, A., Daddi, E., \& Renzini, A. 2006, A\&A, 453, L29

Ciotti, L., Lanzoni, B., \& Renzini, A. 1996, MNRAS, 282, 1

Conroy, C., et al. 2007, ApJ, 654, 153

Croton, D. J., et al. 2006, MNRAS, 365, 11

de Lucia, G., \& Blaizot, J. 2007, MNRAS, 375, 2

di Serego Alighieri, S., et al. 2005, A\&A, 442, 125

Drory, N., Bender, R., \& Hopp, U. 2004, ApJ, 616, L103

Faber, S. M., Dressler, A., Davies, R. L., Burstein, D., \& Lynden-Bell, D. 1987, in Nearly Normal Galaxies: From the Planck Time to the Present, ed. S. M. Faber (New York: Springer), 175

Franceschini, A., et al. 2006, A\&A, 453, 397

Gallazzi, A., Charlot, S., Brinchmann, J., \& White, S. D. M. 2006, MNRAS, 370,1106

Gao, L., White, S. D. M., Jenkins, A., Stoehr, F., \& Springel, V. 2004, MNRAS, 355,819
Gavazzi, R., Treu, T., Rhodes, J. D., Koopmans, L. V., Bolton, A. S., Burles, S., Massey, R., \& Moustakas, L. A. 2007, ApJ, in press (astro-ph/0701589) Graham, A. W., Merritt, D., Moore, B., Diemand, J., \& Terzić, B. 2006, AJ, 132,2701

Kannappan, S. J. \& Gawiser, E. 2007, ApJ, 657, L5

Koopmans, L. V. E., Treu, T., Bolton, A. S., Burles, S., \& Moustakas, L. A. 2006, ApJ, 649, 599

Mandelbaum, R., Tasitsiomi, A., Seljak, U., Kravtsov, A. V., \& Wechsler, R. H. 2005, MNRAS, 362, 1451

Maraston, C., Daddi, E., Renzini, A., Cimatti, A., Dickinson, M., Papovich, C., Pasquali, A., \& Pirzkal, N. 2006, ApJ, 652, 85 (erratum 656, 1241 [2007])

Navarro, J. F., Frenk, C. S., \& White, S. D. M. 1997, ApJ, 490, 493

Padmanabhan, N., et al. 2004, NewA, 9, 329

Pannella, M., et al. 2006, ApJ, 639, L1

Renzini, A. 2006, ARA\&A, 44, 141

Rettura, A., et al. 2006, A\&A, 458, 717

Springel, V., Di Matteo, T., \& Hernquist, L. 2005a, ApJ, 620, L79

Springel, V., et al. 2005b, Nature, 435, 629

Tanaka, M., et al. 2005, MNRAS, 362, 268

Toomre, A. 1977, in Evolution of Galaxies and Stellar Populations, ed. B. M.

Tinsley \& R. B. Larson (New Haven: Yale Univ. Obs.), 401

Treu, T., et al. 2005, ApJ, 633, 174

Trujillo, I., Burkert, A., \& Bell, E. F. 2004, ApJ, 600, L39

van der Wel, A., et al. 2006, ApJ, 652, 97

van Dokkum, P. G. 2005, AJ, 130, 2647

Wirth, G. D., et al. 2004, AJ, 127, 3121 